OPEN ACCESS

Edited by:

Paolo Miccoli,

University of Pisa, Italy

Reviewed by:

Gianlorenzo Dionigi,

University of Milan, Italy

Daniele Barbaro,

UO Endocrinologia ASL nord ovest

Toscana, Italy

*Correspondence:

Xianghui He

hexh88@tmu.edu.cn

Specialty section:

This article was submitted to

Thyroid Endocrinology,

a section of the journal

Frontiers in Endocrinology

Received: 02 November 2021

Accepted: 24 January 2022

Published: 17 February 2022

Citation:

Yan $C$, He $X$, Chen $Z$ and Wang $Y$ (2022) Central Compartment Lymph Nodes Have Distinct Metastatic Patterns in Different Age Groups.

Front. Endocrinol. 13:807431.

doi: 10.3389/fendo.2022.807431

\section{Central Compartment Lymph Nodes Have Distinct Metastatic Patterns in Different Age Groups}

\author{
Caigu Yan ${ }^{1}$, Xianghui He ${ }^{2 *}$, Zuoyu Chen ${ }^{2}$ and Yizeng Wang ${ }^{2}$ \\ ${ }^{1}$ Department of General Surgery, The People's Hospital of Liuyang, Changsha, China, ${ }^{2}$ Department of General Surgery, \\ Tianjin Medical University General Hospital, Tianjin, China
}

Background and Purpose: Central compartment lymph node metastasis (CLNM) is a manifestation of tumor aggressiveness and an indicator of tumor prognosis. The purpose of this study was to construct a nomogram for evaluating CLNM patterns in papillary thyroid carcinoma (PTC) in different age groups.

Method: A total of 907 patients diagnosed with PTC from August 2014 to December 2018 were enrolled. A nomogram illustrating CLNM was generated using the results of multivariate logistic regression analysis.

Results: According to the best Youden index, we set the cut-off age at 45 years. Multivariate logistic regression analysis showed that in patients aged $<45$ years, large tumor size $(P<0.05)$, extra-thyroid extension $(P<0.05)$ and thyroglobulin level $>40 \mathrm{ng} / \mathrm{ml}$ $(\mathrm{OR}=2.985,95 \% \mathrm{Cl}$ 1.379-6.462; $\mathrm{P}<0.05)$ were independent risk factors; meanwhile, Hashimoto's thyroiditis ( $\mathrm{OR}=0.532,95 \% \mathrm{Cl} 0.324-0.874$; $\mathrm{P}<0.05)$ was a protective factor of CLNM. In the subgroup with age $\geq 45$ years, large tumor size $(P<0.05)$, extra-thyroid extension $(\mathrm{P}<0.05)$, unclear margin $(\mathrm{OR}=1.604,95 \% \mathrm{Cl} 1.065-2.416 ; \mathrm{P}<0.05)$, male gender $(\mathrm{OR}=2.009,95 \% \mathrm{Cl} 1.257-3.212 ; \mathrm{P}<0.05)$ were independent risk factors for CLNM. In the subgroup with age $<45$ years, an area under the curve (AUC) of 0.729 (95\% $\mathrm{Cl} 0.680-0.777) ; \mathrm{P}<0.05)$ was obtained. In the $\geq 45$ years subgroup, the AUC was 0.668 (95\% Cl 0.619-0.716; P<0.05).

Conclusion: CLNM of PTC in different age groups may have distinct patterns. Based on the potential risk factors for CLNM in patients with different age stratification, a userfriendly predictive model was established.

Keywords: papillary thyroid carcinoma, central compartment lymph nodes metastasis, age, thyroglobulin, Hashimoto's thyroiditis, prognosis 


\section{BACKGROUND}

Thyroid cancer is becoming one of the most common malignant human tumors. The incidence of thyroid cancer has continued to rise around the world in the past few decades $(1,2)$. Studies have shown that papillary thyroid carcinoma (PTC) has high rates of central compartment lymph nodes metastasis (CLNM), ranging from $20 \%$ to $90 \%$ (3). Prophylactic central lymph node dissection remains controversial (4-6). Some investigators believe that micrometastasis to lymph nodes does not affect the survival time of patients, while other studies have pointed out that prophylactic lymph node dissection could increase the recurrence free-survival rate and reduce postoperative serum thyroglobulin levels, yielding accurate staging to assist further treatment. Moreover, due to the use of nerve monitoring and carbon nanoparticle negative imaging $(7,8)$, permanent damage to the recurrent laryngeal nerve and parathyroid gland is rarely found during dissection of the initial central compartment lymph nodes. Because PTC has a good postoperative survival time, more attention is paid to the probability of postoperative recurrence. when PTC is recurrent and reoperated, it is more likely to cause permanent damage to the recurrent laryngeal nerve and parathyroid gland due to changes in anatomical structure.

CLNM is a manifestation of tumor aggressiveness and an indicator of tumor prognosis. There are many studies assessing the risk factors for CLNM (9-12). However, studies assessing CLNM of thyroid cancer are based on the overall population without age stratification. It is well known that age is a significant factor affecting the biological behavior of thyroid cancer (13). The $8^{\text {th }}$ edition of American Joint Committee on Cancer guidelines indicate elderly patients have decreased disease-free survival (14). Due to different hormone levels and immune states in patients of different ages (15-18), distinct patterns of CLNM may be present.

The purpose of this study was to explore central compartment lymph nodes that have distinct metastatic patterns in different age groups and to assist in clinical decision-making. At the same time, we examined the potential differences in the biological behavior of thyroid cancer in different ages.

\section{MATERIALS AND METHODS}

\section{Clinical Data}

This was a retrospective analysis. A total of 907 patients diagnosed with papillary thyroid carcinoma admitted to a tertiary hospital from August 2014 to December 2018 and operated by the same surgeon were selected as subjects. This study passed the ethical review based on the Declaration of Helsinki, and obtained informed consent from the patients. Preoperatively, all patients underwent fine-needle aspiration cytology (FNA) guided by ultrasound to determine the pathological features, and BRAF V600E was detected at the same time for further diagnosis. Inclusion criteria were: 1) initial thyroid cancer surgery; 2) postoperative pathology confirming thyroid papillary carcinoma; 3) at least unilateral lobectomy plus regional lymph node dissection; 4) completed clinical and pathological data. Exclusion criteria were: 1) recurrent thyroid cancer; 2) incidental thyroid cancer without central lymph node dissection; 3) tumor with lateral cervical lymph node metastasis but no central compartment lymph node metastasis. Figure 1 shows the flow chart of the patients enrolled in this study.
Patients who underwent surgery from
August 2014 toDecember 2018 in our
hospital $(\mathrm{n}=1016)$

Enrolled patients $(\mathrm{n}=907)$

Recurrent thyroid cancer $(\mathrm{n}=20)$

Exclude(n=109) Incidental thyroid cancer without central lymph node dissection( $\mathrm{n}=69$ )

tumor with lateral cervical lymph node metastasis but no central compartment lymph node metastasis $(n=20)$

FIGURE 1 | The flow chart of the patients enrolled in this study. 
All 907 patients underwent standardized surgical treatment, all operations were traditional surgeries rather than endoscopic surgeries. In our center, all patients diagnosed with thyroid papillary carcinoma underwent routine prophylactic central compartment lymph node dissection at least on one side. The surgical methods were as follows: 1) ipsilateral glandular lobe + isthmus resection + unilateral central compartment lymph node dissection (382 cases); 2) ipsilateral glandular lobe + isthmus resection + unilateral central compartment lymph node dissection + lateral neck area dissection (10 cases); 3) total thyroidectomy + unilateral central compartment lymph node dissection + lateral neck area dissection (56 cases); 4) total thyroidectomy + bilateral central compartment lymph node dissection + unilateral neck area dissection (30 cases); 5) total thyroidectomy + bilateral central compartment lymph nodes dissection + bilateral neck area dissection (5 cases); 6) total thyroidectomy + unilateral central compartment lymph nodes dissection (332 cases); 7) total thyroidectomy + bilateral central compartment lymph nodes dissection (92 cases). All surgical methods are shows in Figure 2.

We set the cut-off age at 45 years, which is in line with the age stratification of the $7^{\text {th }}$ edition of the AJCC, and obtained the best Youden index (low false-positive rate or/and high sensitivity). Clinical data included general patient features (gender, age, chief complaint and history of surgery), laboratory data (TSH, FT3,

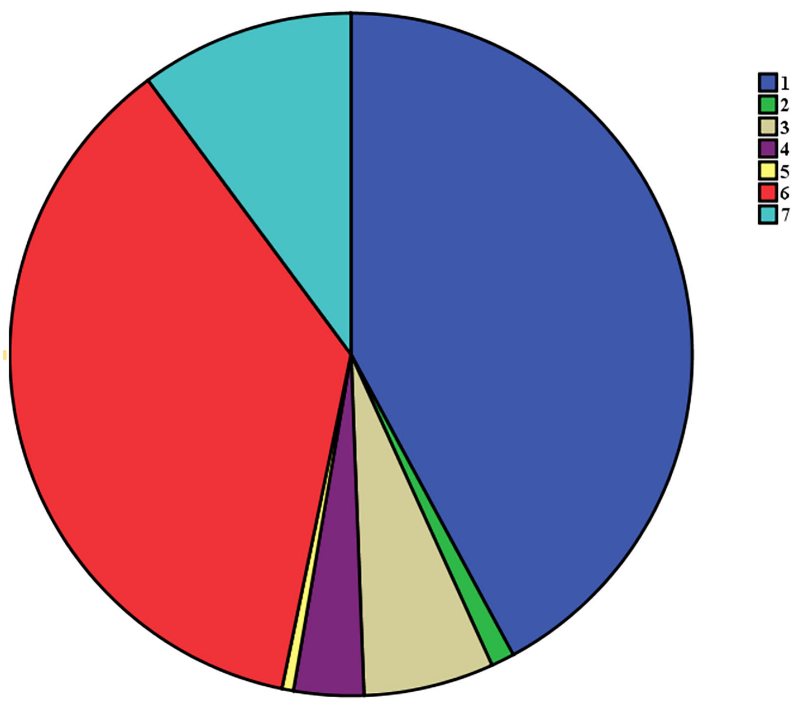

FIGURE 2 | The flow chart of all different surgical procedures:1) ipsilateral glandular lobe + isthmus resection + unilateral central compartment lymph node dissection; 2) ipsilateral glandular lobe + isthmus resection + unilateral central compartment lymph node dissection + lateral neck area dissection; 3) total thyroidectomy + unilateral central compartment lymph node dissection + lateral neck area dissection; 4) total thyroidectomy + bilateral central compartment lymph node dissection + unilateral neck area dissection; 5) total thyroidectomy + bilateral central compartment lymph nodes dissection + bilateral neck area dissection; 6) total thyroidectomy + unilateral central compartment lymph nodes dissection; 7) total thyroidectomy + bilateral central compartment lymph nodes dissection.
FT4, CEA, Tg, Tg-Ab, anti-TPO and PTH), ultrasonographic findings, fine-needle aspiration results, postoperative pathology, length of hospital stay, operation time, intraoperative frozen pathology and final pathological diagnosis. Clinical features were defined as follows: 1)pre-surgical data: gender (male or female), duration of chief complaint (less than 1 year or more than 1 year), aspect ratio (height divided by width on transverse views, $\mathrm{A} / \mathrm{T}$ ), margin on ultrasonogram (clear or unclear), calcification on ultrasonogram (absent or present), TSH (less than $2.7 \mathrm{uIU} / \mathrm{ml}$ or more than $2.7 \mathrm{uIU} / \mathrm{ml} ; 2.7 \mathrm{uIU} / \mathrm{ml}$ is the average value), serum thyroglobulin (less than $40 \mathrm{ng} / \mathrm{ml}$ or more than $40 \mathrm{ng} / \mathrm{ml}$, reference value is less than $40 \mathrm{ng} / \mathrm{ml}$ ), tumor size (less than 1 $\mathrm{cm}, 1 \mathrm{~cm}-2 \mathrm{~cm}$, more than $2 \mathrm{~cm}$ ); 2)post-surgical data: extrathyroidal extension (no capsule contacting, invading capsule and violating surrounding tissues), multifocality (absent or present), bilateral tumor (absent or present), Hashimoto's thyroiditis (absent or present), nodular goiter (absent or present) and CLNM (absent or present). All pathological results were obtained separately by two experienced pathologists; in case of discrepancy, further confirmation was made.

\section{Statistical Analysis}

In this study, the SPSS 22.0 software was used for data analysis. Continuous measurement data were expressed as $\overline{\mathrm{x}} \pm \mathrm{S}$. Univariate logistic regression analysis or chi-square test was performed for various variables. Multivariate analysis was performed by multivariate logistic regression analysis. $\mathrm{P}<0.05$ was considered statistically significant. The test level $\alpha$ was 0.05 on both sides. The process of establishing the nomogram used the R 386 3.5.2 software, using the "rms" software package to construct the nomogram. Through 1000 internal test plots, the calibration curve was compared with the predicted CLNM and actual CLNM rates to evaluate the accuracy of the nomogram. The area under the curve of the ROC curve was plotted according to the predicted CLNM probability to test the diagnostic efficiency.

\section{RESULTS}

\section{Baseline Characteristics and Pathological Features}

Based on clinical features, the 907 subjects were divided as follows: age range of 17-89 years, with 421 younger than 45 years and 486 older than 45 years (average age was $45.6 \pm 12.8$ ); 235 males and 672 females, with a male to female ratio of 1:2.43; 428 (47.2\%) cases had no CLNM and 479 (52.8\%) had CLNM. The remaining features are shown in Table 1.

\section{Single-Factor Analysis of CLNM}

In the $<45$ years subgroup, $270(64.1 \%)$ of all 421 patients had CLNM, versus 209 (43.0\%) of 486 cases older than 45 years old, indicating a statistically significant difference $(\chi 2=39.76$, 
TABLE 1 | Clinicopathological characteristics of the 907 PTC patients.

\begin{tabular}{|c|c|c|c|c|}
\hline \multirow[t]{2}{*}{ Parameter } & \multicolumn{2}{|c|}{$<45$ years $(n=421)$} & \multicolumn{2}{|c|}{$\geq 45$ years $(n=486)$} \\
\hline & No. & Rate (\%) & No. & Rate (\%) \\
\hline \multicolumn{5}{|l|}{ Gender } \\
\hline Male & 130 & 31.9 & 105 & 21.6 \\
\hline Female & 291 & 68.1 & 381 & 78.4 \\
\hline \multicolumn{5}{|l|}{ Hashimoto's thyroiditis } \\
\hline Absent & 296 & 70.3 & 376 & 77.3 \\
\hline Present & 125 & 29.7 & 110 & 22.7 \\
\hline \multicolumn{5}{|l|}{ Tumor size (cm) } \\
\hline$\leq 1$ & 170 & 40.6 & 213 & 43.8 \\
\hline $1-2$ & 180 & 42.8 & 192 & 39.6 \\
\hline$>2$ & 71 & 16.6 & 81 & 16.6 \\
\hline \multicolumn{5}{|l|}{ Multifocality } \\
\hline Absent & 277 & 65.8 & 318 & 65.4 \\
\hline Present & 144 & 34.2 & 168 & 34.5 \\
\hline \multicolumn{5}{|l|}{ Bilateral tumor } \\
\hline Absent & 323 & 76.7 & 379 & 77.9 \\
\hline Present & 98 & 23.3 & 107 & 22.1 \\
\hline \multicolumn{5}{|l|}{ ETE } \\
\hline No capsule contacting & 120 & 28.5 & 117 & 24.0 \\
\hline Invading capsule & 251 & 59.6 & 305 & 62.9 \\
\hline Violating surrounding tissues & 50 & 11.9 & 64 & 13.1 \\
\hline \multicolumn{5}{|l|}{ Duration of chief complaint } \\
\hline$\leq 1$ year & 88 & 20.9 & 354 & 72.9 \\
\hline >1year & 333 & 79.1 & 132 & 27.1 \\
\hline \multicolumn{5}{|l|}{ Margin in ultrasonography } \\
\hline Clear & 233 & 55.3 & 321 & 66.0 \\
\hline Unclear & 188 & 44.7 & 165 & 34.0 \\
\hline \multicolumn{5}{|l|}{ Calcification in ultrasonography } \\
\hline Absent & 109 & 25.9 & 157 & 32.3 \\
\hline Present & 312 & 74.1 & 329 & 67.7 \\
\hline \multicolumn{5}{|l|}{ Aspect ratio } \\
\hline$\leq 1$ & 225 & 53.4 & 241 & 49.5 \\
\hline$>1$ & 196 & 46.6 & 245 & 50.5 \\
\hline TSH & $2.7 \mathrm{ulU} / \mathrm{ml} \pm 5.2$ & & & \\
\hline$\leq 2.7 \mathrm{ulU} / \mathrm{ml}$ & 319 & 75.8 & 325 & 66.8 \\
\hline$>2.7 \mathrm{ulU} / \mathrm{ml}$ & 102 & 24.2 & 161 & 33.2 \\
\hline \multicolumn{5}{|l|}{ Nodular goiter } \\
\hline Absent & 270 & 64.1 & 247 & 50.8 \\
\hline Present & 151 & 35.9 & 239 & 49.2 \\
\hline \multicolumn{5}{|l|}{ Thyroglobulin } \\
\hline$\leq 40 \mathrm{ng} / \mathrm{ml}$ & 350 & 83.1 & 379 & 77.9 \\
\hline$>40 \mathrm{ng} / \mathrm{ml}$ & 71 & 16.9 & 107 & 22.1 \\
\hline \multicolumn{5}{|l|}{ CLNM } \\
\hline Present & 270 & 64.1 & 209 & 43.1 \\
\hline Absent & 151 & 35.9 & 277 & 56.9 \\
\hline
\end{tabular}

PTC, papillary thyroid carcinoma; ETE, extra thyroidal extension; CLNM, central compartment lymph node metastasis.

$\mathrm{P}<0.05)$. Univariate logistic regression analysis showed that in the overall population, gender, age, tumor size, bilateral tumor, degree of capsule invasion, margin on ultrasonogram, calcification on ultrasonogram and serum thyroglobulin had significant associations $(\mathrm{P}<0.05)$ with $\mathrm{CLNM}$, and there were no significant associations of chief complaint duration, multifocality, tumor aspect ratio, TSH, Hashimoto's thyroiditis and nodular goiter with CLNM $(\mathrm{P}>0.05)$. In the $<45$ years subgroup, gender, tumor size, multifocality, bilateral tumor, degree of capsule invasion, Hashimoto's thyroiditis and serum thyroglobulin had significant associations $(\mathrm{P}<0.05)$ with CLNM, and there were no significant associations of chief complaint duration, margin on ultrasonogram, calcification on ultrasonogram, tumor aspect ratio, TSH and nodular goiter with CLNM $(\mathrm{P}>0.05)$. In the $\geq 45$ years subgroup, gender, tumor size, multifocality, bilateral tumor, margin on ultrasonogram and calcification on ultrasonogram had significant associations $(\mathrm{P}<0.05)$ with $\mathrm{CLNM}$. There were no significant differences in the associations of chief complaint duration, tumor aspect ratio, TSH and nodular goiter with CLNM $(\mathrm{P}>0.05)$ (Table 2).

\section{Multi-Factor Analysis of CLNM}

Based on the above univariate analysis, in the overall population, factors that may have associations with CLNM $(\mathrm{P}<0.05)$, 
TABLE 2 | Predictive factors of CLNM in PTC patients according to univariate logistic analysis.

\begin{tabular}{|c|c|c|c|c|c|c|c|}
\hline \multirow[t]{2}{*}{ Parameter } & \multicolumn{3}{|c|}{$<45$ years $(n=421)$} & \multicolumn{3}{|c|}{$\geq 45$ years $(n=486)$} & \multirow{2}{*}{$\frac{\text { Total }}{\mathbf{P}}$} \\
\hline & CLNM(+) $(n=270)$ & CLNM(-) (n = 151) & $\mathbf{P}$ & CLNM(+) (n = 209) & CLNM(-) (n= 277) & $\mathbf{P}$ & \\
\hline Gender & & & 0.006 & & & 0.016 & 0.000 \\
\hline Male & 96 & 34 & & 56 & 49 & & \\
\hline Female & 174 & 117 & & 153 & 228 & & \\
\hline Tumor size $(\mathrm{cm})$ & & & 0.000 & & & 0.000 & 0.000 \\
\hline$\leq 1$ & 91 & 79 & & 70 & 143 & & \\
\hline $1-2$ & 120 & 60 & & 89 & 103 & & \\
\hline$>2$ & 59 & 12 & & 50 & 31 & & \\
\hline Multifocality & & & 0.065 & & & 0.008 & 0.002 \\
\hline Absent & 169 & 108 & & 123 & 195 & & \\
\hline Present & 101 & 43 & & 86 & 82 & & \\
\hline Bilateral & & & 0.051 & & & 0.002 & 0.000 \\
\hline Absent & 199 & 124 & & 149 & 230 & & \\
\hline Present & 71 & 27 & & 60 & 47 & & \\
\hline ETE & & & 0.001 & & & 0.004 & 0.000 \\
\hline No capsule contacting & 60 & 60 & & 36 & 81 & & \\
\hline Capsule invading & 172 & 79 & & 138 & 167 & & \\
\hline Violation in surrounding tissues & 38 & 12 & & 35 & 29 & & \\
\hline Duration of chief complaint & & & 0.515 & & & 0.045 & 0.054 \\
\hline$\leq 1$ year & 53 & 35 & & 162 & 192 & & \\
\hline$>1$ year & 217 & 116 & & 47 & 85 & & \\
\hline Margin of ultrasonography & & & 0.930 & & & 0.012 & 0.011 \\
\hline Clear & 149 & 84 & & 125 & 196 & & \\
\hline Unclear & 121 & 67 & & 84 & 81 & & \\
\hline Calcification in ultrasonography & & & 0.068 & & & 0.015 & 0.001 \\
\hline Absent & 62 & 47 & & 55 & 102 & & \\
\hline Present & 208 & 104 & & 154 & 175 & & \\
\hline Aspect ratio & & & 0.640 & & & 0.424 & 0.604 \\
\hline$\leq 1$ & 142 & 83 & & 108 & 133 & & \\
\hline$>1$ & 128 & 68 & & 101 & 144 & & \\
\hline Nodular goiter & & & 0.085 & & & 0.684 & 0.588 \\
\hline Absent & 165 & 105 & & 104 & 143 & & \\
\hline Present & 105 & 46 & & 105 & 134 & & \\
\hline $\mathrm{TSH}$ & & & 0.795 & & & 0.591 & 0.995 \\
\hline$\leq 2.7 \mathrm{ulU} / \mathrm{ml}$ & 209 & 110 & & 137 & 188 & & \\
\hline$>2.7 \mathrm{ulU} / \mathrm{ml}$ & 61 & 41 & & 72 & 89 & & \\
\hline Thyroglobulin & & & 0.000 & & & 0.661 & 0.012 \\
\hline$\leq 40 \mathrm{ng} / \mathrm{ml}$ & 209 & 141 & & 161 & 218 & & \\
\hline$>40 \mathrm{ng} / \mathrm{ml}$ & 61 & 10 & & 48 & 59 & & \\
\hline Hashimoto's thyroiditis & & & 0.007 & & & 0.555 & 0.354 \\
\hline Absent & 202 & 94 & & 159 & 217 & & \\
\hline Present & 68 & 57 & & 50 & 60 & & \\
\hline
\end{tabular}

including gender, age, tumor size, multifocality, bilateral tumor, degree of capsule invasion, margin on ultrasonogram, calcification on ultrasonogram and serum thyroglobulin, were included in the multivariate logistic regression model. The results showed that large tumor size $(\mathrm{P}<0.05)$, extrathyroidal extension $(\mathrm{P}<0.05)$, male gender $(\mathrm{OR}=1.797,95 \%$ CI 1.288-2.506; $\mathrm{P}<0.05)$, age $<45(\mathrm{OR}=2.426,95 \% \mathrm{CI} 1.818-3.238 ; \mathrm{P}<0.05)$ and calcification $(\mathrm{OR}=1.376,95 \% \mathrm{CI} 1.004-1.885 ; \mathrm{P}<0.05)$ were independent risk factors for CLNM. In the $<45$ years subgroup, factors that may have associations with CLNM $(\mathrm{P}<0.05)$, including gender, tumor size, degree of capsule invasion, Hashimoto's thyroiditis and thyroglobulin, were included in the multivariate logistic regression model. The results showed that large tumor size $(\mathrm{P}<0.05)$, extra-thyroid extension $(\mathrm{P}<0.05)$ and thyroglobulin level $>40 \mathrm{ng} / \mathrm{ml}$ $(\mathrm{OR}=2.985,95 \%$ CI 1.379-6.462; $\mathrm{P}<0.05)$ were independent risk factors for CLNM; meanwhile, Hashimoto's thyroiditis $(\mathrm{OR}=0.532,95 \% \mathrm{CI}$ 0.324-0.874; $\mathrm{P}<0.05)$ were protective factors of CLNM. Gender showed no significant associations ( $P>0.05)$. In the $\geq 45$ years subgroup, factors that may have associations with CLNM $(\mathrm{P}<0.05)$, including gender, tumor size, multifocality, bilateral tumor, margin on ultrasonogram, calcification on ultrasonogram, age, extrathyroidal extension and duration of chief complaint were included in the multivariate logistic regression model. The results showed that large tumor size $(\mathrm{P}<0.05)$, extra-thyroid extension $(\mathrm{P}<0.05)$, unclear margin under ultrasonography $(\mathrm{OR}=1.604,95 \% \mathrm{CI} 1.065-2.416 ; \mathrm{P}<0.05)$, the male gender $(\mathrm{OR}=2.009,95 \% \mathrm{CI} 1.257-3.212$; $\mathrm{P}<0.05)$ were independent risk factors for CLNM. Multifocality, bilateral tumor, calcification on ultrasonogram and duration of chief complaint had no significant associations in multivariate analysis $(\mathrm{P}>0.05)$ (Table 3$)$. 
TABLE 3 | Predictive factors of CLNM in PTC patients in multiple logistic regression analysis.

\begin{tabular}{|c|c|c|c|c|c|}
\hline Parameter & \multicolumn{2}{|c|}{$<45$ years } & \multicolumn{2}{|c|}{$\geq 45$ years } & $\frac{\text { Total }}{\mathbf{P}}$ \\
\hline Age & -- & -- & -- & -- & 0.000 \\
\hline Tumor size $(\mathrm{cm})$ & 0.010 & & 0.000 & & 0.000 \\
\hline $1-2$ & 0.072 & $1.538(0.963-2.457)$ & 0.022 & $1.651(1.077-2.531)$ & \\
\hline$>2$ & 0.004 & $3.122(1.451-6.719)$ & 0.000 & $3.215(1.818-5.683)$ & \\
\hline ETE & 0.003 & & 0.018 & & 0.000 \\
\hline Margin in ultrasonography & -- & -- & 0.024 & $1.604(1.065-2.216)$ & \\
\hline Hashimoto's thyroiditis & 0.010 & $1.901(1.167-3.097)$ & -- & -- & \\
\hline Thyroglobulin & 0.004 & $3.020(1.418-6.432)$ & -- & -- & \\
\hline Calcification & -- & -- & -- & -- & 0.047 \\
\hline Constant & 0.001 & 0.265 & 0.000 & 0.169 & \\
\hline
\end{tabular}

\section{Nomogram Building and Analysis}

Based on the above multivariate logistic regression analysis, a nomogram predictive model was created with the "rms" software package and analyzed with the R software, as shown in Figure 3A, B). Tumor size and extrathyroidal extension are important in CLNM in different age groups. In the $<45$ years subgroup, serum thyroglobulin and extrathyroidal extension accounted for a high weight of CLNM. At the same time, Hashimoto's thyroiditis had a weak protective effect on CLNM. In the $\geq 45$ years subgroup, unclear margin under ultrasonography and male gender were risk factors for CLNM.

After 1000 internal verifications, an internal alignment curve was plotted. In the subgroup with age $<45$ years, the nomogram predictive model showed the average absolute error of the actual risk probability and the predicted risk probability of the model was 0.00016 (Figure 4A). The area under the ROC curve (AUC; Figure 5A, line predmodel 1) was 0.729 (95\% CI 0.680-0.777; $\mathrm{P}<0.05)$, while the AUC based on the overall population was 0.675 (95\% CI 0.623-0.727) (Figure 5A, line predmodel 2). In the subgroup with age $\geq 45$ years, the average absolute error of the actual risk probability and the predicted risk probability of the model was 0.00059 (Figure 4B); the AUC (Figure 5B, line predmodel 3) was 0.668 (95\% CI 0.619-0.716; $\mathrm{P}<0.05)$. The AUC based on the overall population was 0.661 (95\% CI 0.612-0.710; $\mathrm{P}<0.05$ ) (Figure 5B, line predmodel 4).

\section{DISCUSSION}

In the clinical treatment of thyroid papillary carcinoma, there are still many controversies in certain aspects $(19,20)$, including the
A

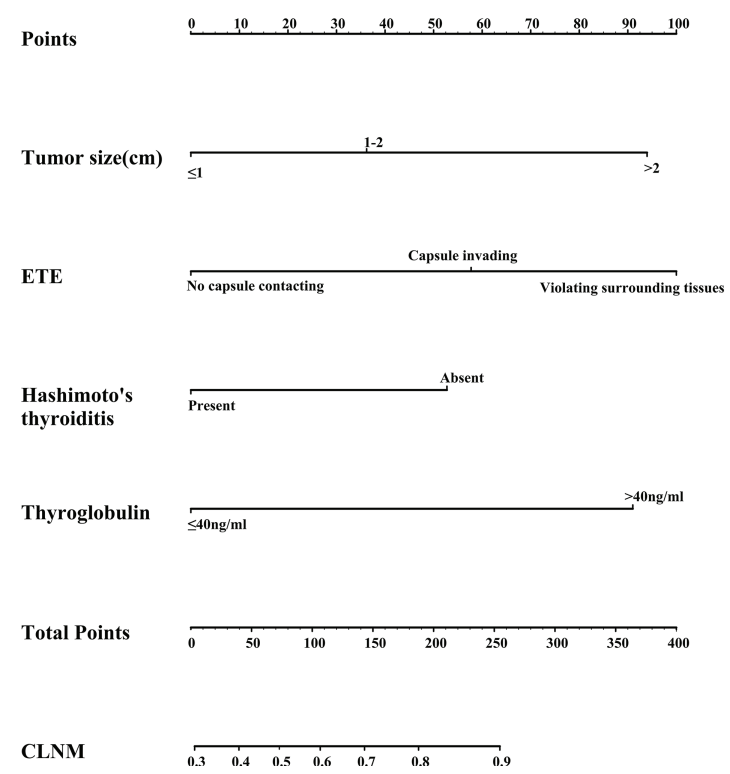

B Points

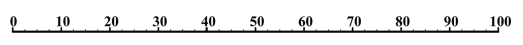
Tumor size(cm) $\quad \leq 1$ 1-2 $>2$ ETE

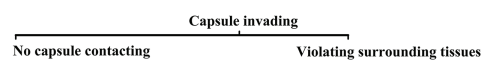

Gender

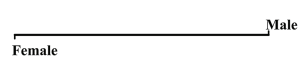

Margin

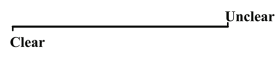

Total Points

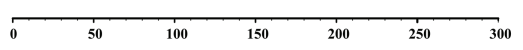

CLNM

FIGURE 3 | (A) Nomogram for predicting CLNM in the subgroup with age $<45$ years; (B) Nomogram for predicting CLNM in the subgroup with age $\geq 45$ years. 
A

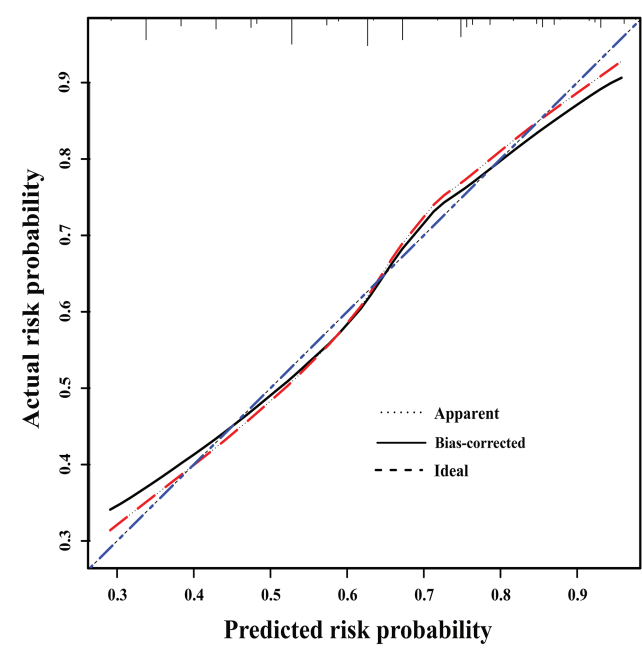

$B=1000$ repetitions, boot

Mean absolute error $=0.00016 n=421$
B

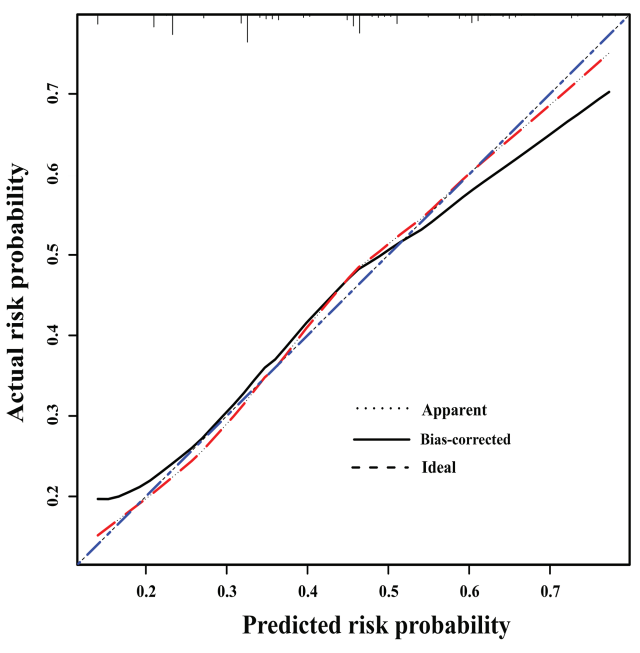

$B=1000$ repetitions, boot

FIGURE 4 | (A) Discrimination plot. The nomogram predictive model showed that in patients <45 years old, the average absolute error of the actual risk probability and the predicted risk probability of this model was 0.00016 . (B) Discrimination plot. The nomogram predictive model showed that in $\geq 45$ years, the average absolute error of the actual risk probability and the predicted risk probability of this model was 0.00059 .

scope of thyroid papillary gland resection and the choice between central and lateral neck prophylactic dissection. In the latest ATA guidelines (20), it was pointed out that in cases with metastasis lymph nodes before the operation, therapeutic central compartment lymph node dissection is recommended, but in cases without central compartment lymph node metastasis before the operation, preventive lymph node dissection is only conducted in progressive disease. The guideline was based on the survival time of patients, not postoperative recurrence, and some data (4) showed that preventive lymph node dissection can reduce the postoperative recurrence of thyroid papillary carcinoma. Because of the shielding of bone and trachea, the detection rate of central compartment lymph nodes by preoperative ultrasound is very low, with a sensitivity of less than $30 \%$ (21). Metastatic lymph nodes in PTC will have the characteristics of increased size, rounded shape, absence of a visible hilum, irregular reflection pattern, unsharp borders, cystic change, calcifications, and nonhilar vasculature $(22,23)$. However, if PTC is complicated with Hashimoto's thyroiditis, we may obtain false-positive results. In our center, all patients diagnosed with thyroid papillary

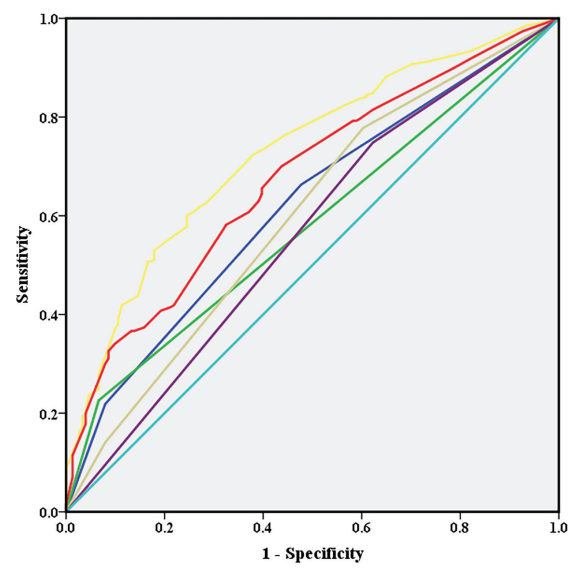

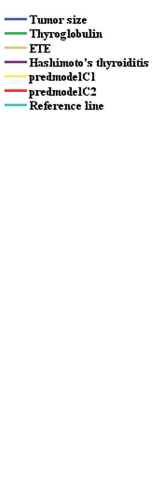

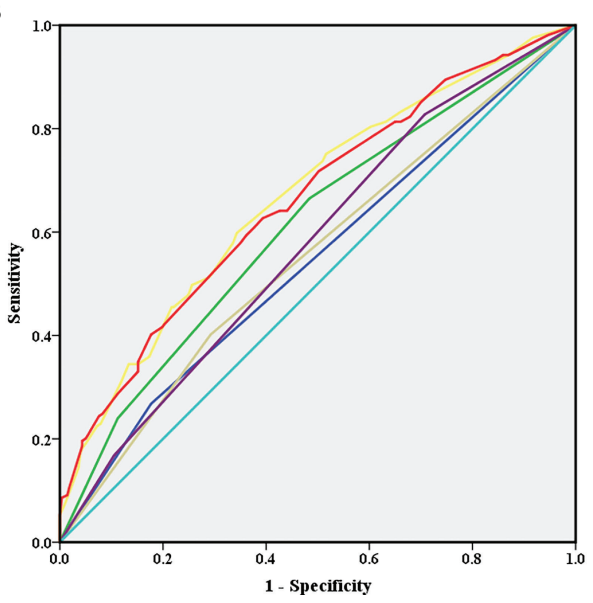

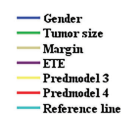

FIGURE 5 | (A) In the subgroup with age <45 years, receiver operating characteristic analysis demonstrated an area under the curve(AUC) of 0.729 (95\% Cl 0.6800.777 ; $\mathrm{P}<0.05)$. (B) In the subgroup with age $\geq 45$ years, receiver operating characteristic analysis demonstrated an $\mathrm{AUC}$ of 0.668 (95\% $\mathrm{Cl} 0.619-0.716$; $\mathrm{P}<0.05)$. 
carcinoma underwent routine prophylactic central compartment lymph node dissection at least on one side. In all of patients, only 21 patients described the abnormal changes of central compartment lymph node at ultrasonography, of which 16 were proved to have CLNM. In the context of this debate, a more sensitive and accurate method for identifying preoperative central compartment lymph nodes becomes important.

Three systematic reviews $(9,11,12)$ of papillary thyroid carcinoma showed gender, age, tumor size, multifocality, capsular invasion, lymphovascular invasion, tumor location, lymphocytic thyroiditis, bilateral tumor, extrathyroidal extension, lateral cervical lymph node metastasis, histological subtype of the tumor and BRAF gene mutations may be risk factors for central compartment lymph node metastasis. There are other studies on proteomics and fibronectin expression in CLNM $(24,25)$. In almost all studies (2628), age, tumor size, gender, multifocality and extrathyroidal extension were considered risk factors for CLNM, but all these reports were based on the overall population without age stratification. It is well-known that age is an important factor affecting the biological behavior of the thyroid, and an important prognostic indicator in thyroid cancer (13). Differentiated thyroid carcinoma is the only tumor with age included in TNM staging. In the $8^{\text {th }}$ edition of AJCC (14), elderly patients are considered to have a higher death risk; however, PTC shows more aggressive biological behavior in younger groups $(29,30)$. Studies have shown that the types of gene mutations in PTC are different in distinct age stratification (31). The importance of age is also reflected in other risk predictive models, including AMES, DAMES and MACIS. In this study, we found that the proportion of CLNM was higher in the younger group, and the proportion of males $(\mathrm{P}<0.05)$ and Hashimoto's thyroiditis incidence $(\mathrm{P}<0.05)$ in younger patients were higher than in older patients.

Based on different age groups, we found that papillary thyroid cancer has different metastatic patterns. In the multifactor study, it was found that in the subgroup with age $<45$ years, tumor size, extrathyroidal extension, Hashimoto's thyroiditis and serum thyroglobulin levels were independent risk factors for CLNM. However, in the subgroup with age $\geq 45$ years, tumor size, extrathyroidal extension, unclear margin under ultrasonography and male gender were independent risk factors for CLNM. Previous studies based on the overall population without age stratification also found that in different studies, factors affecting CLNM are different, which may be caused by the uneven distribution of the population. In this study, as in the overall population, tumor size, extrathyroidal extension, male gender, younger age and calcification were independent risk factors for CLNM. In the present study, we divided the overall population into different subgroups according to age, and the predictive model had a higher area under the ROC curve than the model constructed according to the overall population. Whether this difference is caused by different tumor subtypes or the body's immune status remains to be further studied (15-17). This would enable more accurate clinical decisions in patients of different ages.

In addition, we found that thyroglobulin level was an important factor associated with CLNM in the subgroup with age $<45$ years. In previous studies $(32,33)$, serum thyroglobulin could generally be assessed by lymph node puncture before surgery to determine the status of lymph node metastasis or tumor recurrence after surgery. Besides, previous studies $(27,28)$ have shown that male patients are more likely to have CLNM in PTC, which was reflected in patients older than 45 years, but this trend was not obvious in younger patients. It is well known that differences in sex hormones are more common in younger patients, so the above finding is intriguing. It is worth mentioning that in the younger group, Hashimoto's thyroiditis may be a protective factor of CLNM, but not in older cases. Hashimoto's thyroiditis represents an autoimmune inflammation of the thyroid gland that is common in young patients. Studies have shown that Hashimoto's thyroiditis is a stimulating factor in the occurrence of thyroid papillary carcinoma through immune activation. There are similar findings in existing studies (34-36), with no indication of the role of age.

Our research had limitations as a single-center study with no external validation. Further multi-center studies are required for verifying these findings. In addition, due to the limited number of patients, low AUCs were obtained, and more data are needed to make our results more stable. In addition, the focus of our research is to predict whether there is CLNM, but it cannot be used to predict the number and size of lymph node metastasis, for which we need do further research. Furthermore, long-term follow-up is needed to compare the postoperative recurrence risk of $\mathrm{pN} 1$ patients in different age groups, in order to further clarify the impact of age on tumor metastasis and recurrence.

\section{SUMMARY}

Central compartment lymph nodes of papillary thyroid carcinoma in different age groups may have distinct patterns. The effects of extrathyroidal extension and tumor size in CLNM are reflected in all age groups. In young patients, preoperative serum thyroglobulin levels and the presence of Hashimoto's thyroiditis may affect CLNM, while gender is not significantly important. In older cases, males were more likely to have CLNM. These differences may be explained by different hormone levels and immune states in patients of different ages, which may inform decision-making for individual patients in the clinic.

\section{DATA AVAILABILITY STATEMENT}

The raw data supporting the conclusions of this article will be made available by the authors, without undue reservation.

\section{AUTHOR CONTRIBUTIONS}

$\mathrm{CY}, \mathrm{XH}, \mathrm{ZC}$, and $\mathrm{YW}$ contributed to the conception and design of the study. CY, ZC, and YW organized the database. CY performed the statistical analyses, wrote the first draft of the manuscript, and wrote sections of the manuscript. All authors contributed to manuscript revision and read and approved the submitted version. All authors contributed to the article and approved the submitted version. 


\section{FUNDING}

This work was partially supported by the National Natural Science Foundation of China (81672641).

\section{REFERENCES}

1. Hughes DT, Haymart MR, Miller BS, Gauger PG, Doherty GM. The Most Commonly Occurring Papillary Thyroid Cancer in the United States Is Now a Microcarcinoma in a Patient Older Than 45 Years. Thyroid (2011) 21(3):2316. doi: $10.1089 /$ thy. 2010.0137

2. Morris LGT, Sikora AG, Tosteson TD, Davies L. The Increasing Incidence of Thyroid Cancer: The Influence of Access to Care. Thyroid (2013) 23(7):88692. doi: 10.1089/thy.2013.0045

3. Kim SK, Chai YJ, Park I, Woo J-W, Lee JH, Lee KE, et al. Nomogram for Predicting Central Node Metastasis in Papillary Thyroid Carcinoma. J Surg Oncol (2017) 115(3):266-72. doi: 10.1002/jso.24512

4. Mamelle E, Borget I, Leboulleux S, Mirghani H, Suarez C, Pellitteri PK, et al. Impact of Prophylactic Central Neck Dissection on Oncologic Outcomes of Papillary Thyroid Carcinoma: A Review. Eur Arch Otorhinolaryngol (2015) 272(7):1577-86. doi: 10.1007/s00405-014-3104-5

5. Kutler DI, Crummey AD, Kuhel WI. Routine Central Compartment Lymph Node Dissection for Patients With Papillary Thyroid Carcinoma. Head Neck (2012) 34(2):260-3. doi: 10.1002/hed.21728

6. McHenry CR, Stulberg JJ. Prophylactic Central Compartment Neck Dissection for Papillary Thyroid Cancer. Surg Clin North Am (2014) 94 (3):529-40. doi: 10.1016/j.suc.2014.02.003

7. Randolph GW, Kamani D. Intraoperative Neural Monitoring in Thyroid Cancer Surgery. Langenbecks Arch Surg (2014) 399(2):199-207. doi: 10.1007/ s00423-013-1141-y

8. Wang B, Su AP, Xing TF, Luo H, Zhao WJ, Zhu JQ. The Function of Carbon Nanoparticles to Improve Lymph Node Dissection and Identification of Parathyroid Glands During Thyroid Reoperation for Carcinoma. Med (Baltimore) (2018) 97(32):e11778. doi: 10.1097/MD.0000000000011778

9. Sun W, Lan X, Zhang H, Dong W, Wang Z, He L, et al. Risk Factors for Central Lymph Node Metastasis in CN0 Papillary Thyroid Carcinoma: A Systematic Review and Meta-Analysis. PloS One (2015) 10(10):e0139021. doi: 10.1371/journal.pone.0139021

10. Feng JW, Yang XH, Wu BQ, Sun DL, Jiang Y, Qu Z. Predictive Factors for Central Lymph Node and Lateral Cervical Lymph Node Metastases in Papillary Thyroid Carcinoma. Clin Transl Oncol (2019) 21(11):1482-91. doi: 10.1007/s12094-019-02076-0

11. Ma B, Wang Y, Yang S, Ji Q. Predictive Factors for Central Lymph Node Metastasis in Patients With Cn0 Papillary Thyroid Carcinoma: A Systematic Review and Meta-Analysis. Int J Surg (2016) 28:153-61. doi: 10.1016/j.ijsu.2016.02.093

12. Qu H, Sun GR, Liu Y, He QS. Clinical Risk Factors for Central Lymph Node Metastasis in Papillary Thyroid Carcinoma: A Systematic Review and MetaAnalysis. Clin Endocrinol (Oxf) (2015) 83(1):124-32. doi: 10.1111/cen.12583

13. Wang J, Liu J, Pan H, Jiang C, Liu S, Zhu Z, et al. Young Age Increases the Risk of Lymph Node Positivity in Papillary Thyroid Cancer Patients: A SEER DataBased Study. Cancer Manage Res (2018) 10:3867-73. doi: 10.2147/cmar.S167774

14. Tuttle RM, Haugen B, Perrier ND. Updated American Joint Committee on Cancer/Tumor-Node-Metastasis Staging System for Differentiated and Anaplastic Thyroid Cancer (Eighth Edition): What Changed and Why? Thyroid (2017) 27(6):751-6. doi: 10.1089/thy.2017.0102

15. Nieto H, Boelaert K. WOMEN IN CANCER THEMATIC REVIEW: ThyroidStimulating Hormone in Thyroid Cancer: Does it Matter? Endocr Relat Cancer (2016) 23(11):T109-T21. doi: 10.1530/ERC-16-0328

16. Rinaldi S, Plummer M, Biessy C, Tsilidis KK, Ostergaard JN, Overvad K, et al. Thyroid-Stimulating Hormone, Thyroglobulin, and Thyroid Hormones and Risk of Differentiated Thyroid Carcinoma: The EPIC Study. J Natl Cancer Inst (2014) 106(6):dju097. doi: 10.1093/jnci/dju097

17. Noureldine SI, Tufano RP. Association of Hashimoto's Thyroiditis and Thyroid Cancer. Curr Opin Oncol (2015) 27(1):21-5. doi: 10.1097/CCO.0000000000000150

18. Rahbari R, Zhang L, Kebebew E. Thyroid Cancer Gender Disparity. Future Oncol (2010) 6(11):1771-9. doi: 10.2217/fon.10.127

\section{ACKNOWLEDGMENTS}

I would like to express my special thanks to my partners for the encouragement and support they gave me during my study.

19. Gharib H, Papini E, Garber JR, Duick DS, Harrell RM, Hegedus L, et al. American Association of Clinical Endocrinologists, American College of Endocrinology, and Associazione Medici Endocrinologi Medical Guidelines for Clinical Practice for the Diagnosis and Management of Thyroid Nodules2016 Update. Endocrine Pract (2016) 22:1-60. doi: 10.4158/ep161208.Gl

20. Haugen BR, Alexander EK, Bible KC, Doherty GM, Mandel SJ, Nikiforov YE, et al. American Thyroid Association Management Guidelines for Adult Patients With Thyroid Nodules and Differentiated Thyroid Cancer The American Thyroid Association Guidelines Task Force on Thyroid Nodules and Differentiated Thyroid Cancer. Thyroid (2016) 26(1):1-133. doi: 10.1089/ thy. 2015.0020

21. Sturgeon C, Yang A, Elaraj D. Surgical Management of Lymph Node Compartments in Papillary Thyroid Cancer. Surg Oncol Clinics North America (2016) 25(1):17-40. doi: 10.1016/j.soc.2015.08.013

22. Hwang HS, Orloff LA. Efficacy of Preoperative Neck Ultrasound in the Detection of Cervical Lymph Node Metastasis From Thyroid Cancer. Laryngoscope (2011) 121(3):487-91. doi: 10.1002/lary.21227

23. Lee Y, Kim JH, Baek JH, Jung SL, Park SW, Kim J, et al. Value of CT Added to Ultrasonography for the Diagnosis of Lymph Node Metastasis in Patients With Thyroid Cancer. Head Neck (2018) 40(10):2137-48. doi: 10.1002/ hed. 25202

24. Xia S, Wang C, Postma EL, Yang Y, Ni X, Zhan W. Fibronectin 1 Promotes Migration and Invasion of Papillary Thyroid Cancer and Predicts Papillary Thyroid Cancer Lymph Node Metastasis. Onco Targets Ther (2017) 10:174355. doi: 10.2147/OTT.S122009

25. Lin P, Yao Z, Sun Y, Li W, Liu Y, Liang K, et al. Deciphering Novel Biomarkers of Lymph Node Metastasis of Thyroid Papillary Microcarcinoma Using Proteomic Analysis of Ultrasound-Guided Fine-Needle Aspiration Biopsy Samples. J Proteomics (2019) 204:103414. doi: 10.1016/j.jprot.2019.103414

26. Ahn BH, Kim JR, Jeong HC, Lee JS, Chang ES, Kim YH. Predictive Factors of Central Lymph Node Metastasis in Papillary Thyroid Carcinoma. Ann Surg Treat Res (2015) 88(2):63-8. doi: 10.4174/astr.2015.88.2.63

27. Feng JW, Hong LZ, Wang F, Wu WX, Hu J, Liu SY, et al. A Nomogram Based on Clinical and Ultrasound Characteristics to Predict Central Lymph Node Metastasis of Papillary Thyroid Carcinoma. Front Endocrinol (Lausanne) (2021) 12:666315. doi: 10.3389/fendo.2021.666315

28. Kim SK, Park I, Woo JW, Lee JH, Choe JH, Kim JH, et al. Predictive Factors for Lymph Node Metastasis in Papillary Thyroid Microcarcinoma. Ann Surg Oncol (2016) 23(9):2866-73. doi: 10.1245/s10434-016-5225-0

29. Niemann AC, Reid AT, Smith J, Hammond J, DeBolle SA, Wei I, et al. Association of Patient Age With High-Risk Pathologic Features in Papillary Thyroid Cancer. J Surg Res (2017) 211:228-32. doi: 10.1016/j.jss.2016.12.021

30. Do BA, Payne RJ, Bastianelli M, Mlynarek AM, Tamilia M, Hier M, et al. Is Age Associated With Risk of Malignancy in Thyroid Cancer? Otolaryngol Head Neck Surg (2014) 151(5):746-50. doi: 10.1177/0194599814547503

31. Vriens MR, Moses W, Weng J, Peng M, Griffin A, Bleyer A, et al. Clinical and Molecular Features of Papillary Thyroid Cancer in Adolescents and Young Adults. Cancer (2011) 117(2):259-67. doi: 10.1002/cncr.25369

32. Ritter A, Mizrachi A, Bachar G, Vainer I, Shimon I, Hirsch D, et al. Detecting Recurrence Following Lobectomy for Thyroid Cancer: Role of Thyroglobulin and Thyroglobulin Antibodies. J Clin Endocrinol Metab (2020) 105(6):214551. doi: $10.1210 /$ clinem/dgaa152

33. Darrell C, Nishino M. Thyroglobulin Measurements in Fine Needle Aspiration Specimens From Cervical Lymph Nodes for the Detection of Metastatic Thyroid Cancer: Institutional Analysis of Performance Characteristics. Lab Invest (2020) 100(SUPPL 1):340-1.

34. Donangelo I, Walts AE, Bresee C, Braunstein GD. Lymphocytic Thyroiditis Is Associated With Increased Number of Benign Cervical Nodes and Fewer Central Neck Compartment Metastatic Lymph Nodes in Patients With Differentiated Thyroid Cancer. Endocr Pract (2016) 22(10):1192-8. doi: 10.4158/E151078.OR 
35. Moon S, Chung HS, Yu JM, Yoo HJ, Park JH, Kim DS, et al. Associations Between Hashimoto Thyroiditis and Clinical Outcomes of Papillary Thyroid Cancer: A Meta-Analysis of Observational Studies. Endocrinol Metab (Seoul) (2018) 33(4):473-84. doi: 10.3803/EnM.2018.33.4.473

36. Liang J, Zeng W, Fang F, Yu T, Zhao Y, Fan X, et al. Clinical Analysis of Hashimoto Thyroiditis Coexistent With Papillary Thyroid Cancer in 1392 Patients. Acta Otorhinolaryngol Ital (2017) 37(5):393-400. doi: 10.14639/ 0392-100X-1709

Conflict of Interest: The authors declare that the research was conducted in the absence of any commercial or financial relationships that could be construed as a potential conflict of interest.
Publisher's Note: All claims expressed in this article are solely those of the authors and do not necessarily represent those of their affiliated organizations, or those of the publisher, the editors and the reviewers. Any product that may be evaluated in this article, or claim that may be made by its manufacturer, is not guaranteed or endorsed by the publisher.

Copyright () 2022 Yan, He, Chen and Wang. This is an open-access article distributed under the terms of the Creative Commons Attribution License (CC BY). The use, distribution or reproduction in other forums is permitted, provided the original author(s) and the copyright owner(s) are credited and that the original publication in this journal is cited, in accordance with accepted academic practice. No use, distribution or reproduction is permitted which does not comply with these terms. 